\title{
Structure and dynamics of current sheets at Alfvén resonances in a differentially rotating plasma
}

\author{
X. Wang, A. Bhattacharjee, and Z. W. Ma \\ Department of Physics and Astronomy, University of Iowa, Iowa City, Iowa 52246 \\ C. Ren, C. C. Hegna, and J. D. Callen \\ University of Wisconsin-Madison, 1500 Johnson Drive, Madison, Wisconsin 53706
}

(Received 18 November 1997; accepted 9 January 1998)

\begin{abstract}
Alfvén resonances, where the local flow speed relative to the boundary is equal to the local Alfvén speed, introduce novel dynamical features in a differentially rotating plasma. The spatial structure and dynamics of current sheets in such plasmas is investigated analytically as well as numerically. The current sheets at Alfvén resonances tend to power-law singularities. The growth of current sheets is algebraic in time in the linear regime and saturates in the presence of dissipation without the intervention of nonlinear effects. These results have significant implications for forced reconnection and Alfvén wave dissipation in laboratory and space plasmas. (C) 1998 American Institute of Physics. [S1070-664X(98)00606-5]
\end{abstract}

\section{INTRODUCTION}

The phenomena of current sheet formation and forced reconnection in differentially rotating plasmas are of great interest for space as well as fusion applications. We mention three examples. In a tokamak, forced reconnection caused by resonant field errors can produce current sheets and magnetic islands which can lock to the error field and grow to sufficient size so as to cause disruptions. ${ }^{1}$ In the earth's magnetotail, forced reconnection driven by the solar wind can lead to the formation of thin current sheets observed during the growth and predisruption phase of substorms. ${ }^{2,3}$ In the solar corona, a vacuum magnetic field can be forced by photospheric footpoint motion to form current sheets that can contribute significantly to coronal heating. ${ }^{4}$

It is common practice to describe fundamental features of the phenomena mentioned above by means of a slab model in which the component of the equilibrium magnetic field in the direction of symmetry (the "toroidal" component) is assumed to be a constant, and the magnetic field in the plane perpendicular to this component (the "poloidal" component) changes sign across a surface, known as the neutral line. When a sinusoidal magnetic perturbation is switched on rapidly at the boundary of a stable plasma, thin current sheets tend to grow at the neutral line on the characteristic "poloidal" Alfvén time scale. On a reconnection time scale (which is longer than the Alfvén time scale but shorter than the resistive diffusion time scale), significant magnetic islands develop. The nonlinear dynamics of current sheets and islands driven by such a sinusoidal boundary perturbation, with and without differential rotation, has been the subject of numerous analytical and numerical investigations. $^{5-10}$

The differential rotation can be sustained by various types of momentum sources. The sources can be time independent, as in the tokamak simulations discussed in Ref. 11, where they are centered on flux surfaces, close to the boundaries, on different sides of the neutral line. The simulations are run long enough that they attain steady state. For a given amplitude of the externally imposed magnetic perturbation, two types of steady-state solutions are obtained: those with magnetic islands at the neutral line when the rotation speed is low, and those without islands when the rotation speed is high. ${ }^{8}$ A distinctive feature of the second type of solutions is the development of Alfvén layers (on both sides of the neutral line) where the plasma flow velocity matches the "poloidal" Alfvén speed. ${ }^{11-13}$ These Alfvén layers are the dominant sites where exchange of forces between the plasma and the magnetic field takes place.

A plasma can also be driven by time-dependent, oscillatory momentum sources as, for example, in the coronal Alfvén wave heating simulations discussed in Ref. 14. The objective of these simulations is to study the resonant absorption of Alfvén waves in an inhomogeneous coronal plasma. The initial conditions of these simulations are different from those used in Ref. 11: The plasma is embedded in a shearless magnetic field with an inhomogeneous density profile, and driven at the boundary by an oscillatory forcing function that excites Alfvén waves in the system. As a consequence of strong forcing at the boundary, highly sheared velocity profiles are generated and seen to deform significantly the ideal resonant heating layer ${ }^{15,16}$ In particular, resonant heating is seen to occur dominantly via current sheets in two narrow layers around the ideal Alfvén resonance layer, qualitatively similar to the Alfvén layers seen in Ref. 11.

The main focus of the present paper is on the structure and dynamics of near-singular current sheets at Alfvén resonances in a differentially rotating plasma. We consider the physically interesting case of a sheared background flow that cannot be eliminated by a Galilean transformation. Such a flow can be realized either by moving the boundaries with the plasma at rest, or by moving the plasma in between fixed boundaries. While both cases are equivalent and can be treated by the methodology presented in this paper, for 
specificity we treat the first case. Alfvén resonances can occur in such configurations at the surface where the flow velocity relative to the moving boundary matches the local Alfvén speed. Previous theoretical work has focused on the strong suppression of magnetic islands at the neutral line under these conditions, but the structure and dynamics of the current sheets at the Alfvén resonances have not been considered in sufficient detail.

The plan of this paper is as follows. In Sec. II, we present the model and equations that enable us to treat current sheets at Alfvén resonances in systems with sheared magnetic fields as well as stratified density profiles. Our objective is to obtain basic results that are applicable to systems such as tokamaks as well as the solar corona. In Secs. III and IV, we discuss, respectively, the linear and the nonlinear phases of current sheet growth, and verify the analytical results by numerical simulation. We conclude in Sec. V with a summary and a brief discussion of the possible implications of our results.

\section{MODEL AND EQUATIONS}

We use the geometry and coordinates of Taylor's model. ${ }^{6,8-10,12}$ The initial magnetic field is given by $\mathbf{B}_{0}$ $=\hat{y} B_{0} x / a+\hat{z} B_{T}$ for $|x| \leqslant a$, where $B_{0}, B_{T}$, and $a$ are constants. There is a neutral line at $x=0$. Assuming translation symmetry along $z$ and ignoring perturbations of the $z$ component of $\mathbf{B}_{0}$, we represent the time-dependent magnetic field as $\mathbf{B}=\hat{z} \times \nabla \psi(x, y, t)+\hat{z} B_{T}$, where $\psi$ is a flux function. For tokamak applications (such as Ref. 11), the initial flux is given by $\psi_{0}=B_{0} x^{2} / 2 a$. Later, for coronal applications (such as Ref. 14), we take $\psi_{0}=B_{0} x$ and $B_{T}=0$.

As discussed in Sec. I, we will consider the case in which the boundaries at $x= \pm a$ are moved suddenly with a constant velocity $\mathbf{u}_{0}=\hat{y} V_{0}$. For the coronal application, $V_{0}$ is the phase velocity of the photospheric driver, oscillating with frequency $\omega_{0} \equiv V_{0} / k$. The viscous relaxation of the fluid velocity inside the plasma will be governed by the momentum equation which, in the presence of the time-dependent inertial term, is an equation of the diffusion type. The velocity profile will show a tendency to relax to the uniform state $\mathbf{u}_{0}=\hat{y} V_{0}$ on a viscous diffusion time scale, $\tau_{V}=\rho a^{2} / \nu$ (where $\rho$ is the density and $\nu$ is the coefficient of viscosity) through a sequence of intermediate states $\mathbf{u}_{0}=\hat{\mathbf{y}} u_{0}(x, t)$. In many low-collisionality systems of physical interest, the viscous relaxation time is much longer than the nonlinear reconnection time. Hence for dynamics on the ideal magnetohydrodynamic (MHD) and reconnection time scales, we can assume $\mathbf{u}_{0}=\hat{y} u_{0}(x)$ and suppress the slow time dependence of the background shear flow.

We now perturb the background plasma by a smallamplitude sinusoidal perturbation at the moving boundary, represented by $x= \pm\left[a-\xi_{0} \cos \left(k y-k V_{0} t\right)\right]$ where $\left|\xi_{0} / a\right|$ $\ll 1$. As a result of this perturbation, the plasma exhibits dynamics on a much faster time scale than the slow viscous relaxation time of the background flow. We assume that the time-dependent perturbed plasma velocity $\mathbf{v}$ lies entirely in the $(x, y)$ plane and represent it as $\mathbf{v}=\hat{\mathbf{z}} \times \nabla \phi(x, y, t)$, where $\phi$ is a stream function. The two-dimensional MHD equations can then be written (in cgs units) as

$$
\begin{aligned}
& \frac{\partial \psi}{\partial t}+\mathbf{v} \cdot \nabla \psi=\frac{\eta c^{2}}{4 \pi} \nabla^{2} \psi \\
& \rho\left[\frac{\partial \nabla^{2} \phi}{\partial t}+\mathbf{v} \cdot \nabla \nabla^{2} \phi\right]=\hat{z} \cdot\left[\nabla \psi \times \nabla\left(\nabla^{2} \psi\right)\right] / 4 \pi+\nu \nabla^{4} \phi
\end{aligned}
$$

We rewrite $\xi_{0} \cos \left(k y-k V_{0} t\right)$ as the real part of $\xi_{0} \exp [i(k y$ $\left.\left.-k V_{0} t\right)\right]$ and express the linear perturbations in the form

$$
\begin{aligned}
& \psi_{1}(x, y, t)=\tilde{\psi}(x, t) \exp \left[i\left(k y-k V_{0} t\right)\right], \\
& \phi_{1}(x, y, t)=\tilde{\phi}(x, t) \exp \left[i\left(k y-k V_{0} t\right)\right] .
\end{aligned}
$$

The linearized ideal equations for the outer regions are

$$
\begin{aligned}
& {\left[\frac{\partial}{\partial t}+i k \Delta V(x)\right] \tilde{\psi}=i k \tilde{\phi} B_{0 y}(x),} \\
& {\left[\frac{\partial}{\partial t}+i k \Delta V(x)\right]\left[\nabla^{2} \tilde{\phi}+\frac{\rho^{\prime}}{\rho} \tilde{\phi}^{\prime}\right]} \\
& \quad-i k \tilde{\phi}\left[\Delta V^{\prime \prime}(x)+\frac{\rho^{\prime}}{\rho} \Delta V^{\prime}(x)\right]=i k \frac{B_{0 y}(x)}{\rho} \nabla^{2} \tilde{\psi},
\end{aligned}
$$

where $\Delta V(x) \equiv u_{0}(x)-V_{0}$ is the difference between the plasma flow velocity and the velocity of the moving boundary, $\nabla^{2} \equiv \partial^{2} / \partial x^{2}-k^{2}$ is the two-dimensional Laplacian, and the prime indicates spatial derivative. Note that although the velocities $u_{0}(x)$ and $V_{0}$ vary from one Galilean reference frame to another, the relative velocity $\Delta V(x)$ is frame invariant.

We assume that the leading-order, outer-region solution is quasisteady in time. Let $\rho_{0}$ be the density and $V_{A 0}$ $=B_{0} / \sqrt{4 \pi \rho_{0}}$ the poloidal Alfvén speed at $x=a$. With the redefinition $\rho / \rho_{0} \rightarrow \rho, \tilde{\psi} / B_{0} a \rightarrow \tilde{\psi}\left(B / B_{0} \rightarrow B\right), \tilde{\phi} / V_{A 0} a \rightarrow \tilde{\phi}$, $k a \rightarrow k, x / a \rightarrow x$, and $\alpha \equiv \Delta V(x) / V_{A 0}$, we obtain the following dimensionless equations for the outer region:

$$
\begin{aligned}
& \alpha \tilde{\psi}=B_{0 y} \tilde{\phi} \\
& \alpha\left[\nabla^{2} \tilde{\phi}+\frac{\rho^{\prime}}{\rho} \tilde{\phi}^{\prime}\right]-\left[\alpha^{\prime \prime}(x)+\frac{\rho^{\prime}}{\rho} \alpha^{\prime}(x)\right] \tilde{\phi}=\frac{B_{0 y}}{\rho} \nabla^{2} \tilde{\psi} .
\end{aligned}
$$

Note that if the normalized velocity difference $\alpha$ is a global constant, it must be zero everywhere because of the boundary condition $\alpha( \pm a)=0$. In that case, Eqs. (3a) and (3b) reduce, respectively, to the outer region equations for Taylor's model, $\widetilde{\phi}=0$ and $\nabla^{2} \widetilde{\psi}=0$, and the Alfvén resonances do not appear. Hence differential rotation is an essential ingredient for the new physical effects discussed in this paper. (Although $\alpha$ is not constant everywhere, it can, of course, have local regions of flatness.)

We first consider a plasma of constant density, embedded in a magnetic field with a neutral line (i.e., $B_{0 y}=x$ ), appropriate for tokamak applications. Equation (3a) yields $\widetilde{\psi}=x \widetilde{\phi} / \alpha$, which can be substituted in Eq. (3b) to obtain 


$$
\begin{gathered}
\left(x^{2}-\alpha^{2}\right) \tilde{\phi}^{\prime \prime}+2 x\left(1-x \alpha^{\prime} / \alpha\right) \tilde{\phi}^{\prime}+\left[k^{2}\left(\alpha^{2}-x^{2}\right)-\frac{2 \alpha^{\prime} x}{\alpha}\right. \\
\left.+\frac{2 \alpha^{\prime 2} x^{2}}{\alpha^{2}}-\frac{\alpha^{\prime \prime} x^{2}}{\alpha}+\alpha \alpha^{\prime \prime}\right] \tilde{\phi}=0 .
\end{gathered}
$$

A notable feature of Eq. (4) is the presence of two Alfvén resonances at $x= \pm \alpha$, or $x= \pm \Delta V(x) / V_{A 0}$. In general, if we define $V_{A}(x) \equiv B_{0 y}(x) / \sqrt{4 \pi \rho(x)}$, the Alfvén resonances obey the relation $\Delta V(x)= \pm V_{A}(x)$. Near these resonances, it is clear from Eq. (4) that the vorticity $\widetilde{\omega} \equiv \widetilde{\phi}^{\prime \prime}$ exhibits singular behavior. Since $\tilde{\phi}^{\prime \prime} \rightarrow \infty$ as $x \rightarrow \pm \alpha$, we conclude from the relation $\tilde{\psi}=x \tilde{\phi} / \alpha$ that $\tilde{\psi}^{\prime \prime} \rightarrow \widetilde{\phi}^{\prime \prime}$ in this limit and hence, the current density $\tilde{j} \equiv \widetilde{\psi}^{\prime \prime}$ must also be singular at the Alfvén resonances.

We carry out a local analysis near the singular points of Eq. (4) to determine the spatial structure of the outer region solutions. Let $x_{0}$ be a positive root of the algebraic equation $x^{2}-\alpha^{2}(x)=0$. In the neighborhood of $x=x_{0}$, the velocity profile in general can be approximated by a linear expansion in $x$. Hence we write

$$
\alpha(x) \approx \alpha\left(x_{0}\right)+\alpha^{\prime}\left(x_{0}\right)\left(x-x_{0}\right)=x_{0}+\Omega_{0} \theta,
$$

where $\alpha^{\prime}\left(x_{0}\right) \equiv \Omega_{0}, \theta \equiv x-x_{0} \equiv \epsilon \theta_{0}$ and $\epsilon$ is a small parameter. As $x \rightarrow x_{0}(\theta \rightarrow 0)$, to leading order in $\epsilon$, Eq. (4) yields,

$$
\theta_{0} \frac{\partial F^{(0)}}{\partial \theta_{0}}+F^{(0)}=0
$$

where $F \equiv \widetilde{\phi}^{\prime}$ and the superscript zero indicates the leadingorder solution. Equation (5) shows that the leading-order perturbed velocity has a power-law singularity, i.e., $F^{(0)} \sim \theta_{0}^{-1}$ $\sim\left(x-x_{0}\right)^{-1}$. It follows that the stream function $\phi^{(0)}$ has a logarithmic singularity. However, the vorticity $\widetilde{\phi}^{\prime \prime(0)} \sim \theta_{0}^{-2}$ $\sim 1 /\left(x-x_{0}\right)^{2}$ has a stronger power-law singularity, and so does the current sheet $\widetilde{\psi}^{\prime \prime(0)}$. This power-law singularity of the current density should be contrasted with the $\delta$-function singularity obtained at the neutral line in Taylor's model.

We next consider the structure of the singular outer region solutions for coronal Alfvén wave heating applications, discussed, for instance in Ref. 14, where the density is stratified but the magnetic field is shearless. We take $B_{0 y}=1$, and obtain from Eqs. (3a) and (3b),

$$
\begin{aligned}
& \left(1-\rho \alpha^{2}\right) \tilde{\phi}^{\prime \prime}-\left(\rho^{\prime} \alpha^{2}+2 \alpha^{\prime} / \alpha\right) \tilde{\phi}^{\prime} \\
& \quad+\left[k^{2}\left(\rho \alpha^{2}-1\right)+\frac{2 \alpha^{\prime 2}}{\alpha^{2}}-\frac{\alpha^{\prime \prime}}{\alpha}+\alpha\left(\rho \alpha^{\prime \prime}+\rho^{\prime} \alpha^{\prime}\right)\right] \tilde{\phi}=0 .
\end{aligned}
$$

The singular points of Eq. (6), and hence, the resonant layers are located where $1-\rho \alpha^{2}=0$. Recalling the definition $V_{A}(x) \equiv B_{0 y} / \sqrt{4 \pi \rho(x)}$, we can rewrite this resonance condition as $\left(k u_{0}-\omega_{0}\right)^{2}=k^{2} V_{A}^{2}$ or $\Delta V(x)= \pm V_{A}(x)$, which is identical to the resonance condition in the first case. When $u_{0}(x)=0$, we obtain the standard Alfvén wave resonance condition $\omega_{0}^{2}=k^{2} V_{A}^{2}$. For given continuous profiles $\rho(x)$ and $u_{0}(x)$, there are at least two shifted resonance layers, specified by the relation $\omega_{0}=k u_{0} \pm k V_{A}$.
As in the case with the neutral line, we can expand around the shifted resonances to write $\rho \alpha^{2} \equiv 1+\Omega \theta$ $+O\left(\theta^{2}\right)$, which defines $\Omega$. It follows that

$$
\rho^{\prime} \alpha^{2}+2 \alpha^{\prime} / \alpha=\left(\rho \alpha^{2}\right)^{\prime}-2 \alpha^{\prime} \alpha \rho\left[1-\frac{1}{\rho \alpha^{2}}\right]=\Omega+O(\theta),
$$

which, when substituted in Eq. (6), yields Eq. (5) again. Therefore the structure of the singular outer region solution in this case is similar to that in the first case.

It should be noted that the singular structure of the ideal perturbed velocity (or $\tilde{\phi}^{\prime}$ ) obtained from Eq. (5) is different from that of the standard Alfvén resonance. ${ }^{15-19}$ Whereas the perturbed velocity has a logarithmic singularity near the resonance in the standard case, it has a power-law singularity, scaling as $\left(x-x_{0}\right)^{-1}$, near the flow-driven Alfvén resonance.

\section{LINEAR DYNAMICS OF THE CURRENT SHEET}

\section{A. The linear ideal phase}

The time development of current sheets at Alfvén resonances can be determined by a high-order inner region calculation, using the asymptotic behavior of the outer solution $F^{(0)} \sim \theta_{0}^{-1} \sim\left(x-x_{0}\right)^{-1}$ as the matching condition. For the Alfvén resonance layer of width $\sim O(\epsilon)$ we introduce a slow time scale $t_{0}=\epsilon t$ so that $\partial / \partial t=\epsilon \partial / \partial t_{0}$. At leading order, Eq. (2) yields

$$
\widetilde{\psi}^{(0)}=\widetilde{\phi}^{(0)}, \quad \widetilde{\omega}^{(0)}=\tilde{j}^{(0)} .
$$

At $O(\epsilon)$, we obtain

$$
\begin{aligned}
& {\left[\frac{\partial}{\partial t_{0}}-i k\left(1-\Omega_{0}\right) \theta_{0}\right] \widetilde{\phi}^{(0)}=i k x_{0}\left[\widetilde{\phi}^{(1)}-\widetilde{\psi}^{(1)}\right],} \\
& {\left[\frac{\partial}{\partial t_{0}}-i k\left(1-\Omega_{0}\right) \theta_{0}\right] \widetilde{\omega}^{(0)}=-i k x_{0}\left[\widetilde{\phi}^{(1)}-\widetilde{\psi}^{(1)}\right]^{\prime \prime},}
\end{aligned}
$$

where the superscript one denotes the first-order solution. Eliminating the first-order terms between Eqs. (8a) and (8b), we obtain

$$
\left[\theta_{0}+\frac{i}{k\left(1-\Omega_{0}\right)} \frac{\partial}{\partial t_{0}}\right] \frac{\partial F^{(0)}}{\partial \theta_{0}}+F^{(0)}=0 .
$$

We introduce the Laplace transform $\bar{f}\left(\theta_{0}, p_{0}\right)$ $\equiv \int_{0}^{\infty} d t_{0} e^{-p_{0} t_{0}} f\left(\theta_{0}, t_{0}\right)$ and transform Eq. (9) to

$$
\left[p_{0}-i k\left(1-\Omega_{0}\right) \theta_{0}\right] \frac{\partial \bar{F}^{(0)}}{\partial \theta_{0}}=i k\left(1-\Omega_{0}\right) \bar{F}^{(0)} \text {. }
$$

By Eq. (7), the current density is given by $\bar{j}^{(0)}=\bar{\omega}^{(0)}$ $=\partial \bar{F}^{(0)} / \partial \theta_{0}$. Differentiating Eq. (10), we obtain the equation for the current density

$$
\left[p_{0}-i k\left(1-\Omega_{0}\right) \theta_{0}\right] \frac{\partial \bar{j}^{(0)}}{\partial \theta_{0}}=2 i k\left(1-\Omega_{0}\right) \bar{j}^{(0)} .
$$

Integrating Eq. (11) and then taking the limit $\theta_{0} \rightarrow \infty$, which corresponds to the outer limit of the inner region, we obtain

$$
\bar{j}^{(0)}(0) \approx-\left[\frac{k\left(1-\Omega_{0}\right) \theta_{0}}{p_{0}}\right]^{2} \bar{j}^{(0)}\left(\theta_{0} \rightarrow \infty\right) .
$$


Matching the outer limit of the inner region to the inner limit of the outer region, we obtain

$$
\bar{j}_{\text {inner }}^{(0)}\left(\theta_{0} \rightarrow \infty\right)=\bar{j}_{\text {outer }}^{(0)}\left(x \rightarrow x_{0}\right)=\frac{\Psi_{0}}{\left(x-x_{0}\right)^{2} p_{0}}=\frac{\Psi_{0}}{\epsilon^{2} \theta_{0}^{2} p_{0}},
$$

where $\Psi_{0} \sim B_{0} \xi_{0}$. From Eqs. (12) and (13) we obtain

$$
\bar{j}^{(0)}(0) \approx-\frac{k^{2}\left(1-\Omega_{0}\right)^{2} \Psi_{0}}{\epsilon^{2} p_{0}^{3}} .
$$

Thus the current density at the Alfvén resonances $x= \pm x_{0}$ or $\theta_{0}=0$ is given by

$$
\tilde{j}(0)=-k^{2}\left(1-\Omega_{0}\right)^{2} \Psi_{0} t_{0}^{2} / \epsilon^{2}=-k^{2}\left(1-\Omega_{0}\right)^{2} \Psi_{0} t^{2},
$$

which grows algebraically with time.

We note that Eq. (9) is invariant under the transformations $\theta_{0} \rightarrow \beta \theta_{0}, t \rightarrow \beta^{-1} t$ where $\beta$ is a constant. ${ }^{6}$ While the width of this ideal current sheet scales as $\theta_{0}^{-2}$, its amplitude grows as $t^{2}$ until dissipation intervenes. As shown below, resistivity and/or viscosity then slows down the ideal dynamics and saturates the growth of the current sheet.

\section{B. The linear nonideal phase}

The linearized nonideal MHD equations can be written as

$$
\begin{aligned}
& \left(\frac{\partial}{\partial t}+i k \alpha\right) \tilde{\psi}-i k x \tilde{\phi}=\frac{1}{S} \tilde{\psi}^{\prime \prime}, \\
& \left(\frac{\partial}{\partial t}+i k \alpha\right) \tilde{\phi}^{\prime \prime}-i k \alpha^{\prime \prime} \tilde{\phi}=i k x \tilde{\psi}^{\prime \prime}+\frac{1}{R} \widetilde{\phi}^{\prime \prime \prime \prime},
\end{aligned}
$$

where $S \equiv \tau_{R} / \tau_{A}$ is the Lundquist number, $R \equiv \tau_{\nu} / \tau_{A}$ is the Reynolds number, $\tau_{R} \equiv 4 \pi a^{2} / \eta c^{2}$, and $\tau_{\nu} \equiv \rho a^{2} / \nu$.

We consider the case $S \ll R$ first. Resistivity dominates as a dissipation mechanism in this case, and the effect of viscosity can be neglected. Resistive effects enter the induction equation when $S \epsilon^{2} \partial / \partial t \sim O(1)$ for $\partial / \partial t \sim O(\epsilon)$, which shows that the width of the Alfvén layer scales as $\epsilon$ $=S^{-1 / 3} \cdot{ }^{20}$ Hence we redefine $t_{0} \equiv t / \tau_{L}=\epsilon t$ (where $\tau_{L}$ $\left.=S^{1 / 3} \tau_{A}\right)$ and write $\partial / \partial t=\epsilon \partial / \partial t_{0}$. To zeroth order in $\epsilon$, we obtain Eq. (7). The $O(\epsilon)$ equations are

$$
\begin{aligned}
& {\left[\frac{\partial}{\partial t_{0}}-i k\left(1-\Omega_{0}\right) \theta_{0}\right] \tilde{\phi}^{(0)}-\widetilde{\omega}^{(0)}=i k x_{0}\left[\widetilde{\phi}^{(1)}-\widetilde{\psi}^{(1)}\right],} \\
& {\left[\frac{\partial}{\partial t_{0}}-i k\left(1-\Omega_{0}\right) \theta_{0}\right] \widetilde{\omega}^{(0)}=-i k x_{0}\left[\widetilde{\phi}^{(1)}-\widetilde{\psi}^{(1)}\right]^{\prime \prime} .}
\end{aligned}
$$

Eliminating the first-order terms between Eqs. (17a) and (17b), we obtain

$$
\left[\theta_{0}+\frac{i}{k\left(1-\Omega_{0}\right)} \frac{\partial}{\partial t_{0}}\right] \frac{\partial F^{(0)}}{\partial \theta_{0}}+F^{(0)}=\frac{i}{2 k\left(1-\Omega_{0}\right)} \frac{\partial^{3} F^{(0)}}{\partial \theta_{0}^{3}} .
$$

The Laplace transform of Eq. (18) yields

$$
\left[p_{0}-i k\left(1-\Omega_{0}\right) \theta_{0}\right] \frac{\partial \bar{F}^{(0)}}{\partial \theta_{0}}-i k\left(1-\Omega_{0}\right) \bar{F}^{(0)}=\frac{1}{2} \frac{\partial^{3} \bar{F}^{(0)}}{\partial \theta_{0}^{3}} .
$$

In the limit $t \ll \tau_{L}$ or $p_{0} \gg 1$, Eq. (19) reduces to Eq. (10). We thus recover the ideal current sheet (15). [In this limit the right-hand side of Eq. (18) can be shown to be negligible. $\left.{ }^{6}\right]$ It is interesting to note that although resistivity determines the characteristic time scale $\tau_{L}$, it does not appear explicitly in the expression for the current sheet amplitude. ${ }^{6,9}$ In other words, the current sheet amplitude grows at the same ideal rate independent of the level of dissipation, although the time scale over which the ideal growth persists is determined by the dissipation.

In the limit $t \gg \tau_{L}$ or $p_{0} \ll 1$, the current sheet becomes sufficiently thin that the ordering $\epsilon \sim S^{-1 / 3}$ breaks down. Then the resistive term in Eq. (16a) becomes much larger than $O(\epsilon)$ and the equations need to be reordered. A consistent ordering can be found for $\epsilon=S^{-1 / 2}$, with redefined $t_{0}$ $\equiv t / \tau_{S P}=\epsilon t\left(\tau_{S P}=S^{1 / 2} \tau_{A}\right)$ which implies $\partial / \partial t=\epsilon \partial / \partial t_{0}$. In this asymptotic limit the contribution due to the current sheet is sufficiently large that it changes the leading-order equation (7) to

$$
\widetilde{\psi}^{(0)}=\widetilde{\phi}^{(0)}-\frac{i}{k x_{0}} \widetilde{j}^{(0)}, \quad \widetilde{\omega}^{(0)}=\widetilde{j}^{(0)} .
$$

From Eq. (20) it follows that a quasisteady solution with a current sheet is attainable in the linear regime and obeys the condition $\left[\tilde{j}^{(0)}\right]^{\prime \prime}=0$. In the limit $t \gg \tau_{S P}$, or $\partial / \partial t_{0} \sim p_{0} \ll 1$, the quasisteady current sheet is given by $\widetilde{j}^{(0)} \approx \widetilde{j}^{(0)}(0)$ $=$ constant.

In the case with $S \gg R$, we have $R \epsilon^{2} \partial / \partial t \sim O(1)$. Since $\partial / \partial t \sim O(\epsilon)$, we obtain $\epsilon=R^{-1 / 3}$. Then the leading-order solutions continue to obey Eq. (7), and the next-order solutions are determined by the equations

$$
\begin{aligned}
& {\left[\frac{\partial}{\partial t_{0}}-i k\left(1-\Omega_{0}\right) \theta_{0}\right] \widetilde{\phi}^{(0)}=i k x_{0}\left[\widetilde{\phi}^{(1)}-\widetilde{\psi}^{(1)}\right],} \\
& {\left[\frac{\partial}{\partial t_{0}}-i k\left(1-\Omega_{0}\right) \theta_{0}\right] \widetilde{\omega}^{(0)}=\left[\widetilde{\omega}^{(0)}\right]^{\prime \prime}-i k x_{0}\left[\widetilde{\phi}^{(1)}-\widetilde{\psi}^{(1)}\right]^{\prime \prime} .}
\end{aligned}
$$

It is easy to verify that Eqs. (21a) and (21b) also lead to Eq. (19). Thus for $t \ll R^{1 / 3}$, we again obtain the "ideal" current sheet (15). Note, however, that the quasisteady current sheet is determined, not by Eq. (20), but by

$$
\widetilde{\psi}^{(0)}=\widetilde{\phi}^{(0)}, \quad \widetilde{\omega}^{(0)}=\tilde{j}^{(0)}-\frac{i}{k x_{0}}\left[\widetilde{\omega}^{(0)}\right]^{\prime \prime},
$$

with $\epsilon=R^{-1 / 2}$. Equations (22) imply that the quasisteady current sheet obeys

$$
\left[\widetilde{\omega}^{(0)}\right]^{\prime \prime}=0, \quad\left[\widetilde{j}^{(0)}\right]^{\prime \prime}=0 .
$$

Thus the quasisteady current sheet is given by $\widetilde{j}^{(0)} \approx \widetilde{j}^{(0)}(0)$ $=$ constant, as in the case $S \ll R$. 


\section{THE NONLINEAR REGIME}

It is interesting to note that, according to the discussion in Sec. III, the rate of growth of the current sheet slows down within the realm of validity of linear theory without the intervention of nonlinear effects. We now demonstrate that as long as the magnitude of the sinusoidal boundary perturbation remains small, the current sheets at the Alfvén resonance remain essentially in the linear regime. In such a plasma, the magnitude of the Eulerian displacement $\xi$ from a stable MHD equilibrium is bounded from above by the maximum size $\xi_{0}$ of the boundary perturbation. The magnitude of the perturbed flow $\tilde{\mathbf{u}}=\partial \xi / \partial t$ scales as $\xi / \tau$ where $\tau$ is the characteristic time scale of the perturbation. At the Alfvén resonance $x=x_{0}$, the flow velocity $u_{0}$ relative to the boundary is equal to the local Alfvén speed $V_{A}\left(x_{0}\right)=V_{A} x_{0} / a=x_{0} / \tau_{A}$. Hence, we obtain

$$
\frac{\tilde{u}}{u_{0}}<\frac{\xi_{0} \tau_{A}}{x_{0} \tau}=\frac{\epsilon \xi_{0}}{x_{0}},
$$

where $\epsilon=S^{-1 / 3}, S^{-1 / 2}\left(\epsilon=R^{-1 / 3}, R^{-1 / 2}\right)$ for the resistive (viscous) case. If $\epsilon \xi_{0} \ll x_{0}$, the plasma dynamics near the Alfvén resonance remains in the linear regime. It is now easy to check that this inequality is indeed satisfied if the flowdriven Alfvén resonances are well-separated from the neutral line (or the standard Alfvén resonance, $\omega_{0}^{2}=k^{2} V_{A}^{2}$ ). To realize well-separated flow-driven Alfvén resonances, we must have $x_{0}>\Delta \sim \epsilon a$, where $\Delta$ is the width of the inner layer near the neutral line (or the standard Alfvén resonance). Since $\xi_{0} \ll a$, it follows that the condition $x_{0}>\Delta \sim \epsilon a \gg \epsilon \xi_{0}$ must hold.

In the case with the neutral line, if the condition $x_{0}>\Delta$ $\sim \epsilon a \gg \epsilon \xi_{0}$ does not apply, the flow-driven Alfvén resonances will not be sufficiently well-separated from the inner layer at the neutral line. Then the dynamics will be similar to that in Taylor's model which exhibits a current sheet as well as magnetic islands at the neutral line. ${ }^{6,9,10}$ We point out that whereas nonlinear effects are very important for the current sheet and reconnection dynamics in Taylor's model, ${ }^{9}$ they are not so for the saturation of current sheets at the flowdriven Alfvén resonances.

The analytical calculations described above have been tested by a two-dimensional MHD simulation. (For a more detailed discussion of the computer code, the reader is referred to Ref. 10.) In these simulations, the nonlinear equations (1) are integrated forward in time with a sinusoidal boundary perturbation with the initial analytical equilibrium $\psi_{0}=B_{0} x^{2} / 2 a$ which has a neutral line at $x=0$. The parameters of the simulation are $\xi_{0}=5 \sqrt{\pi} 10^{-4}, \quad k a=\pi / 2$, $V_{0}=0.2 V_{A}, S=10^{5}$, and $R=10^{4}$. In Fig. 1 we show the contour plots for the current density $J_{z}$ in the $x-y$ plane. Two distinct current sheets are seen, coincident with the flowdriven Alfvén layers, on two opposite sides of the neutral line. Neither a current sheet nor significant islands are seen to form near $x=0$, consistent with the results obtained in Refs. 11 and 13. In Fig. 2 we show the time evolution of the maximum current sheet amplitude near one of the Alfvén resonances. The solid line indicates the output from the nonlinear code and the dotted line is the output from the code

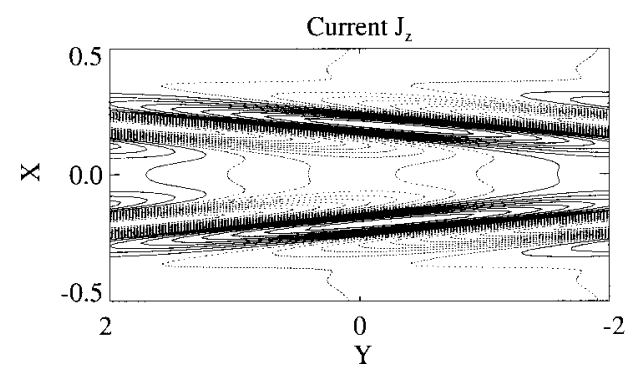

FIG. 1. Contour plot of the current density $J_{z}$ showing current sheets at the Alfvén layers $\left(t=200 \tau_{A}\right)$.

which integrates the linearized equations. After a short-lived transient phase during which the sinusoidal perturbation is switched on to its full value, ${ }^{10}$ both codes show that the current sheets at the Alfvén resonances grow algebraically with time (as indicated by the dot-dashed line), consistent with Eq. (15). The linear and nonlinear codes show saturation of the current sheet amplitude at comparable levels, supporting our claim that nonlinearities do not play an essential role in determining the saturation level. As the magnitude of $V_{0}$ is reduced, the Alfvén layers approach the neutral line and coalesce with it for low differential speeds. ${ }^{10,13}$

Current sheets, qualitatively similar to the ones shown in Fig. 1, are also seen in the coronal Alfvén wave heating simulations of Ref. 14. (See Plates 1-4 of Ref. 14.)

\section{SUMMARY AND DISCUSSIONS}

In a differentially rotating, resistively stable plasma driven by a sub-Alfvénic boundary perturbation, a singularity occurs at Alfvén resonances where the frame-invariant flow speed difference $(\Delta V)$ between the plasma and the boundary matches the local Alfvén speed. The suppression of magnetic reconnection at the neutral line under such conditions has been discussed elsewhere. ${ }^{11-13}$ We have focused here instead on the structure and dynamics of current sheets at the Alfvén resonances. The current sheets tend to be power-law singularities near the Alfvén resonances, unlike the $\delta$-function singularity near the neutral line when the differential rotation is sufficiently small. ${ }^{6-10}$ We have calculated analytically and verified by numerical simulation the time development of these current sheets which grow algebra-

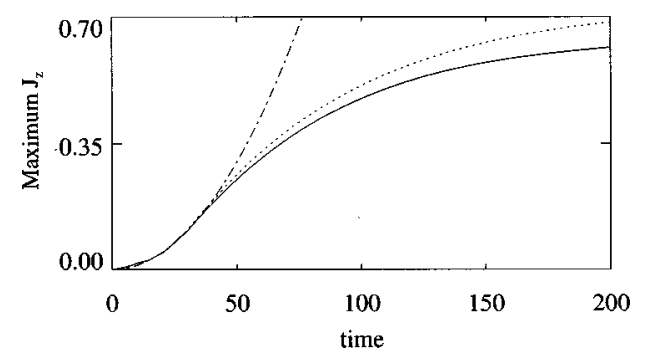

FIG. 2. The growth and saturation of the maximum current sheet amplitude at the Alfvén layers as a function of time (measured in units of $\tau_{A}$ ). The solid and dotted lines represent the outputs from the nonlinear and linear codes, respectively. The dot-dashed line is a theoretical fit and is consistent with Eq. (15). 
ically with time in the early linear regime and saturate in the presence of dissipation without the intervention of nonlinear effects.

The current sheets at Alfvén layers experience torques that tend to slow down the plasma and bring these layers closer to the neutral line. In order to keep these layers wellseparated from the neutral line and thus suppress the growth of islands, it will be necessary to sustain the differential rotation profile of the plasma by momentum input from external sources. In tokamaks, this will require substantial and sustained momentum input, for instance, by neutral beams. While this may be feasible in small tokamaks, it is unlikely to be practical for reactor-grade, large tokamaks.

We have also applied the present model to Alfvén wave propagation in coronal plasmas in which the density is stratified but the magnetic field is shearless. If the velocity driver at the boundary is made large, the standard Alfvén resonance is replaced by flow-driven Alfvén resonances. We have shown that the current sheets at these flow-driven resonances have a double-pole structure, which is more singular than the current sheet at the standard Alfvén resonance. As discussed in Refs. 21-23 and simulated in Ref. 14, hydrodynamic instabilities can be excited in such systems and enhance the dissipation at the resonance layers. Whenever such shear layers occur, flow-driven Alfvén resonances should play a significant role in the linear as well as nonlinear resonant heating of coronal plasmas. For low Lundquist numbers $(S$ $\leqslant 10^{3}$ ), scaling laws for the velocity fluctuations and the coronal heating rate have been obtained numerically in Ref. 14. Analytical and numerical results on the relevant scaling relations in the high-Lundquist-number regime are left to future work.

\section{ACKNOWLEDGMENTS}

This research is supported by the National Science Foundation Grant Nos. ATM 93-10157 and ATM 95-28598 and the U.S. Department of Energy Grant No. DE-FG0286ER53218.

\footnotetext{
${ }^{1}$ See, for instance, R. J. La Haye, R. Fitzpatrick, T. C. Hender, A. W. Morris, J. T. Scoville, and T. N. Todd, Phys. Fluids B 4, 2098 (1992), and other references therein.

${ }^{2}$ S. Ohtani, K. Takahashi, L. J. Zanetti, T. A. Potemra, R. W. McEntire, and T. Iijima, J. Geophys. Res. 97, 19311 (1992).

${ }^{3}$ The observations reported in Ref. 2 have been simulated by Z. W. Ma, X. Wang, and A. Bhattacharjee, Geophys. Res. Lett. 22, 21 (1995).

${ }^{4}$ See, for instance, E. N. Parker, Spontaneous Current Sheets in Magnetic Fields (Oxford, New York, 1994), and other references therein.

${ }_{5}^{5}$ W. Park, D. A. Monticello, and R. B. White, Phys. Fluids 27, 137 (1984).

${ }^{6}$ T. S. Hahm and R. M. Kulsrud, Phys. Fluids 28, 2412 (1985).

${ }^{7}$ F. L. Waelbroeck, Phys. Fluids B 1, 2372 (1989).

${ }^{8}$ R. Fitzpatrick and T. C. Hender, Phys. Fluids B 3, 644 (1991).

${ }^{9}$ X. Wang and A. Bhattacharjee, Phys. Fluids B 4, 1795 (1992).

${ }^{10}$ Z. W. Ma, X. Wang, and A. Bhattacharjee, Phys. Plasmas 3, 2427 (1996).

${ }^{11}$ O. A. Hurricane, T. H. Jensen, and A. B. Hassam, Phys. Plasmas 2, 1976 (1995).

${ }^{12}$ C. Ren, C. C. Hegna, and J. D. Callen, Bull. Am. Phys. Soc. 41, 1371 (1996).

${ }^{13}$ A. H. Boozer, Phys. Plasmas 3, 4602 (1996).

${ }^{14}$ L. Ofman and J. M. Davila, J. Geophys. Res. 100, 23427 (1995).

${ }^{15}$ A. Hasegawa and L. Chen, Phys. Rev. Lett. 32, 454 (1974).

${ }^{16}$ J. A. Tataronis, J. Plasma Phys. 13, 87 (1975).

${ }^{17}$ Y. Mok and G. Einaudi, J. Plasma Phys. 33, 199 (1985).

${ }^{18}$ J. V. Hollweg, Astrophys. J. 312, 880 (1987).

${ }^{19}$ J. M. Davila, Astrophys. J. 317, 514 (1987).

${ }^{20}$ J. M. Kappraff and J. A. Tataronis, J. Plasma Phys. 18, 209 (1977).

${ }^{21}$ J. Heyvaerts and E. R. Priest, Astron. Astrophys. 117, 220 (1983).

${ }^{22}$ P. K. Browning and E. R. Priest, Astron. Astrophys. 131, 283 (1984).

${ }^{23}$ J. V. Hollweg and G. Yang, J. Geophys. Res. 93, 5423 (1988).
} 